BULL. AUSTRAL. MATH. SOC.

$05 \mathrm{C9}, 60499,62 \mathrm{N99}, 90 \mathrm{BI}$

VOL. $29(1984), 139-140$.

\title{
DisCRETE GREEN'S FUNCTIONS AND RANDOM RESISTIVE NETWORKS
}

\section{Cezary S, Surma}

This thesis studies deterministic and random networks by investigating electrical networks composed solely of resistors with, respectively, deterministic and random resistances. The former are called the resistive networks, the latter the random resistive networks.

An important network - theoretical problem of expressing global network parameters in terms of local network parameters is addressed to. For the resistive networks explicit formulae are obtained giving resistance between any two nodes, or sets of nodes, in terms of the resistances of the deterministic component resistors. For the random resistive networks moments, for example the variance, of the (random) resistance between any two nodes, or sets of nodes, are explicitly found in terms of the moments of the resistances of the random component resistors.

Also, the concept of the discrete Green's function of a network is proposed and networks are considered in terms of their discrete Green's functions. A number of properties of the discrete Green's functions of networks are introduced and proved. They are used to construct for each network a new distance function via the network's discrete Green's function. Such a metric, unlike the conventional network metrics, is characteristic of each network, and for the case of a resistive network is shown to give the distance between any two nodes as equal to the value of

Received 2 November 1983. Thesis submitted to Australian National University, July 1983. Degree approved October 1983. Supervisor: Professor P.A.P. Moran.

Copyright Clearance Centre, Inc. Serial-fee code: 0004-9727/84 $\$$ A2.00 + 0.00 . 
the resistance between those nodes. Thus, electrical resistance is postulated to be a metric on resistive networks.

A closed method of finding explicit formulae for the discrete Green's functions of a wide class of networks is presented, and some specific resistive and random resistive networks of interest are studied in detail after their discrete Green's functions are found explicitly by the introduced method. Simulation techniques are also used on those specific networks to confirm the theoretical results.

Possible applications of the obtained results to the following related areas are indicated: random walks, percolation theory, network sensitivity analysis, network optimization, transportation networks, transport phenomena in random media, theory of infinite networks.

Department of Mathematics, Faculty of Applied Science, Royal Melbourne Institute of Technology, GPO Box 2476V, Mel bourne, Victoria 3001 , Australia. 